\title{
General Psychiatry Efficacy of repetitive transcranial magnetic stimulation in treatment- resistant depression: the evidence thus far
}

\author{
Aditya Somani, ${ }^{1}$ Sujita Kumar Kar ${ }^{\odot 2}$
}

To cite: Somani A, Kar SK. Efficacy of repetitive transcranial magnetic stimulation in treatment-resistant depression: the evidence thus far. General Psychiatry 2019;32:e100074. doi:10.1136/ gpsych-2019-100074

Received 29 March 2019 Revised 05 June 2019 Accepted 13 June 2019
Check for updates

(C) Author(s) (or their employer(s)) 2019. Re-use permitted under CC BY-NC. No commercial re-use. See rights and permissions. Published by BMJ.

${ }^{1}$ Department of Psychiatry, Mental Health Institute, Chandigarh, India ${ }^{2}$ Department of Psychiatry, King George's Medical University, Lucknow, Uttar Pradesh, India

Correspondence to Dr Sujita Kumar Kar; drsujita@gmail.com

\section{ABSTRACT}

Depression is a common mental disorder, which attributes to significant morbidity, disability and burden of care. A significant number of patients with depression still remain symptomatic after adequate trials of antidepressant treatment as well as psychotherapy, which is often referred to as treatment-resistant depression. Neuromodulation techniques-like electroconvulsive therapy, vagus nerve stimulation, transcranial magnetic stimulation (TMS) and transcranial direct current stimulation, may be useful augmenting techniques in depression, mostly recommended for treatment-resistant cases. Robust evidence exists regarding the efficacy of electroconvulsive therapy in the management of treatment-resistant depression; however, other techniques are understudied. TMS has been increasingly studied in various psychiatric disorders including depression. It has been approved by the US Food and Drug Administration for use in major depressive disorder. Over the past two decades, TMS has been studied in diverse groups of the population with depression using several research designs. This article gives an overview of the efficacy of repetitive TMS in treatment-resistant depression with the recent evidence.

\section{INTRODUCTION}

Depression is a common mental disorder that affects more than 300 million people worldwide. ${ }^{1}$ Depression is a leading cause of morbidity and its contribution to global burden of diseases is likely to increase with time. It affects people of all ages, races, gender and communities. It is a significant public health concern. ${ }^{2}{ }^{3}$ Effective treatment of depression is available in the form of drugs, psychotherapy, electroconvulsive therapy (ECT) and other non-invasive brain stimulation methods. However, prevalence of treatment resistance in depression is close to $20 \%$ and research scholars are looking for options to tackle this challenge. ${ }^{34}$

Transcranial magnetic stimulation (TMS) is one of the newer options to treat depression. $^{5-7}$ The technique of TMS was first developed by Barker and his colleagues in
$1985 .^{8}$ Subsequently, researchers came up with modalities to deliver multiple pulses in a short interval which came to be known as repetitive TMS (rTMS) ${ }^{5}$ Thereafter, rTMS has come a long way and is now being used or investigated for its potential therapeutic use in numerous psychiatric and physical disorders. ${ }^{67}$ Two major modalities of rTMS used in clinical practice are 'high frequency rTMS' $(\geq 1 \mathrm{~Hz})$ and 'low frequency rTMS' $(\leq 1 \mathrm{~Hz})$. High-frequency rTMS is thought to have a stimulating effect on the cerebral cortex, whereas low-frequency rTMS is thought to have an inhibitory effect. ${ }^{79} \mathrm{~A}$ newer modality of rTMS is theta burst stimulation (TBS) in which relatively greater amounts of stimulation can be delivered to the brain in a shorter span of time. ${ }^{10} 11 \mathrm{~A}$ standard rTMS session takes around $30 \mathrm{~min}$ whereas TBS session takes only $3 \mathrm{~min}$ and the latest research finds this modality to be non-inferior to high-frequency rTMS when used in patients with treatment-resistant depression. ${ }^{12}$

Essentially, rTMS has earned itself a special place in the treatment of depression across the world and a lot of research is being focused over the same. Research findings suggest that there is asymmetry in the functioning of the frontal lobe in patients with depression. ${ }^{13}$ Based on this concept, researchers have tried to treat patients with inhibitory (low frequency) stimulation to the right dorsolateral prefrontal cortex (DLPFC) or excitatory (high frequency) stimulation to the left DLPFC. ${ }^{1415}$ Nearly a decade ago, the use of TMS was approvedby the US Food and Drug Administration (US FDA) for treatment-resistant depression. A specific protocol (stimulatory protocol delivering 3000 pulses per session over the right DLPFC) has been approved by the US FDA; however, over the past decade various 
other protocols as well as different TMS techniques have been tried. More evidence has been gathered through comparison of efficacy of TMS with pharmacotherapy and ECT. This accumulating evidence gives a better insight to the efficacy of TMS in treatment-resistant depression as an augmenting therapeutic modality. This review shall discuss the efficacy of rTMS in the management of treatment-resistant depression and other related aspects as per current literature.

\section{Efficacy of rTMS in treatment-resistant depression}

Mostly, rTMS has been studied as an adjunct to pharmacotherapy. Kedzior et al have done a systematic review and meta-analysis of 16 double-blind, sham-controlled randomised controlled trials (RCT) using high-frequency rTMS over the left DLPFC. ${ }^{16}$ The antidepressant effect of rTMS was studied in follow-up and it was found that patients with unipolar depression, less severe depressive episodes, treatment-resistant depression, non-psychotic depression and those who were on concomitant antidepressant therapy had a better antidepressant effect with rTMS. ${ }^{16}$ In their systematic review and meta-analysis, Berlim et al evaluated double-blind, randomised, sham-controlled trials of low-frequency rTMS in major depression. ${ }^{17}$ In this meta-analysis, the patients with major depressive disorder had some degree of treatment resistance. It was found that patients with major depression receiving low-frequency rTMS as monotherapy had significant reduction of depressive symptoms in comparison to the sham controls. Patients receiving $>1200$ pulses in total often had good efficacy in terms of response as well aspression. The autho remission. ${ }^{17}$ Berlim et al have also carried out a detailed analysis of data on the use of high-frequency rTMS for treatment of depression. ${ }^{18}$ After the analysis of data from 29 RCTs covering 1371 patients, they found that $29.3 \%$ of patients responded to treatment and $18.6 \%$ of patients could achieve remission with high-frequency rTMS. The OR for the pooled data was 3.3 with the $p$ value less than 0.0001 for response and remission. ${ }^{18}$ In this meta-analysis, again out of 29 RCTs, 18 had patients with treatment-resistant depression. The authors reported that there was a difference in terms of efficacy of high-frequency rTMS in treatment-resistant depression versus a lesser degree of treatment resistance.

Researchers have compared and analysed if there is any difference in the efficacy of rTMS applied to left versus right DLPFC. ${ }^{19}$ In their analysis, Chen et al pooled the data from eight RCTs and found that both high and low-frequency rTMS applied to left and right DLPFC, respectively, were equally effective. However, incidence of side effects, including seizures, was lesser with low-frequency right-sided rTMS. $^{19}$

Gaynes et al focused their meta-analysis on the role of rTMS in treatment-resistant depression..$^{20}$ They searched the literature and evaluated 18 studies that rTMS was compared with sham-controlled treatment in patients who had failed to respond to two or more antidepressants. They could conclude with a high strength of evidence that rTMS produced a meaningful decrease in Hamilton Depression
Rating Scale (HDRS) and likelihood to response was more than three times when compared with patients on sham control. Furthermore, patients on rTMS were five times more likely to achieve remission. ${ }^{20}$ In a latest meta-analysis by Wei $e t$ al, results from 29 studies covering a total sample of 1659 subjects were aggregated. ${ }^{21}$ The authors found that rTMS worked well in combination with antidepressants, especially when used for treatment-resistant or refractory depression. Important parameters that affected treatment were intensity of stimulus, frequency of stimulus train, site of stimulation and course of treatment. ${ }^{21}$ One should note that this meta-analysis had a significant representation of data from Chinese studies (10 out of 29 studies, 572 out of 1659 cases were from China).

One meta-analysis from Canada did not find rTMS as effective as described in the studies mentioned above. ${ }^{22}$ The authors pooled the data from 23 RCTs that compared rTMS with sham controls. The primary outcome was change in scores of HDRS and they had set a mean difference of 3.5 points to be clinically significant. Pooled data showed rTMS to have a statistically significant improvement in depression scores but the mean difference in scores of HDRS in two groups was 2.32, although less than the predecided cut-off. Therefore, the authors concluded that rTMS had only a small, short-term effect in improving treatment-resistant depression. ${ }^{22}$

Few authors have also tried bilateral rTMS, that is, sequential administration of low-frequency rTMS in the right DLPFC followed by high-frequency rTMS in the left DLPFC in patients with treatment-resistant depression. ${ }^{23}$ Initial research showed a better remission rate in patients getting bilateral rTMS in comparison to the sham group. In the same paper, remission rate in the group of patients getting unilateral rTMS did not differ either from bilateral rTMS or the sham group. ${ }^{23}$ However, the meta-analysis by Zhang et al gives a different finding. ${ }^{24}$ Data from 10 RCTs that had assessed 634 patients were evaluated. It was found that bilateral rTMS was significantly more effective than sham rTMS (risk ratio $=3.43, \mathrm{p}<0.001$ ). When compared with unilateral rTMS, the scores were only marginally high (risk ratio $=1.01$, $\mathrm{p}=0.93$ ) and did not reach the level of statistical significance. The authors concluded that bilateral rTMS was not better than unilateral rTMS and might not be useful for patients with treatment-resistant depression. ${ }^{24}$ Berlim et al had also concluded similar findings in their meta-analysis that there was no significant difference in efficacy and acceptability between bilateral and unilateral rTMS in treatment-resistant depression. ${ }^{25}$ In a recent study, Stubbeman et a $t^{26}$ used bilateral $20 \mathrm{~Hz}$ TBS using the neuronavigation technique in treatment-resistant depression and found the response rate to be $72 \%{ }^{26}$ However, such TMS protocols were innovations in the treatment of treatment-resistant depression and were not routinely followed. There is a need to evaluate the relevance of such therapeutic innovations in larger populations.

TMS is also found to be effective in bipolar depression. Deep TMS (dTMS) has been used in the treatment of bipolar depression and the early results suggest 
that the depressive features improve with TMS. ${ }^{27} 28$ The therapeutic response in patients receiving true TMS is better than those receiving sham TMS at the end of therapy. Patients receiving maintenance TMS sessions have better response than those not receiving the maintenance sessions. ${ }^{27}{ }^{28}$ However, in a recent study, both low and high-frequency rTMSs given with concomitant quetiapine treatment in patients with bipolar-II depression were not found to be better in comparison to quetiapine monotherapy either in terms of symptom resolution or cognitive side effects over a 4-week trial period. ${ }^{29}$ Very recently, newer TMS modalities have been used in treatment-resistant depression. Low-frequency magnetic stimulation (low-intensity magnetic waveform), which is known to modulate spontaneous neuronal oscillation and metabolism without altering the action potential of the neuron, is found to have a mood-enhancing effect in treatment-resistant depression. ${ }^{30}$ This process is less time consuming than the conventional TMS procedures. Further research in a larger population is needed to evaluate its therapeutic potential in treatment-resistant depression. Similarly, accelerated protocol of rTMS (more frequent sessions like more than one session per day) has been tried in patients with treatment-resistant depression. The authors found that accelerated protocols of intermittent TBS and high-frequency $(20 \mathrm{~Hz})$ rTMS are equally effective in treatment-resistant depression. ${ }^{31}$ Accelerated protocols curtail the duration of TMS treatment and are expected to produce early symptom reduction; hence worth studying in future research. In their open-label study on patients with unipolar and bipolar depression, Schulze $e t a l^{32}$ found an accelerated protocol (two sessions per day) to be equally effective as the conventional TMS session that is once a day. ${ }^{32}$ Most existing studies evaluate the role of TMS as an augmenting modality to antidepressant treatment. There is a paucity of literature that focuses on the augmenting role of TMS to psychotherapy in patients with treatment-resistant depression. A recent study evaluated the efficacy of simultaneous rTMS and psychotherapy in depression in a naturalistic setting. A total of 196 patients diagnosed with major depressive disorder were included in the study, out of which more than $97 \%$ were having treatment resistance. The response rate and remission rate at the end of combined psychotherapy and TMS treatment were $66 \%$ and $56 \%$, respectively. ${ }^{33}$ It indicates that TMS can be used as an augmenting agent to psychotherapy in treatment-resistant depression with good chances of remission.

\section{rTMS versus ECT in treatment-resistant depression}

The place of ECT in the treatment of severe depression, psychotic depression, depression with self-harm or treatment-resistant depression is well known and does not need any elaboration or reference. ECT remains the treatment of choice in all patients where there is a need for early response because of the risk of harm to self or catatonia. No discussion on the efficacy of rTMS in depression shall be meaningful without discussing its comparison with ECT. Hansen $e t$ al compared low-frequency rTMS applied to the right DLPFC with ECT in 60 patients under a randomised study design. ${ }^{34}$ The rate of partial remission was higher by $26 \%$ in the ECT group. However, patients receiving ECT had more cognitive side effects compared with those receiving rTMS. ${ }^{34}$ In this study, most patients had severe depression and most of them were treatment non-responders (treatment-resistant depression). In the meta-analysis by Berlim et al, data from seven RCTs were pooled and it was found that $52 \%$ of patients receiving ECT achieved remission whereas only $33 \%$ of those receiving rTMS achieved remission. ${ }^{35}$ At the same time, there was no difference in the rate of dropouts in both the treatment groups. The authors reached a conclusion that ECT appeared to be more effective than rTMS in the treatment of depression. This review had exclusively compared ECT with high-frequency rTMS. ${ }^{35}$ Again most of the patients included in this meta-analysis were having treatment resistance at baseline. Another meta-analysis with a larger patient base compared both low and high-frequency rTMSs with ECT. ${ }^{36}$ ECT was found to be superior to rTMS in terms of both response and remission. Superiority of ECT was more pronounced in patients with psychotic depression. However, in the same analysis, high-frequency rTMS was found to be equally effective as ECT in non-psychotic depression. Acceptability of both the modalities was comparable in short term. As one would expect, this study also showed that cognitive side effects (in domains of visual memory and verbal fluency) were more common in patients receiving ECT. ${ }^{36}$ Looking at the level of evidence available currently, it appears that ECT shall continue to hold its place in treating patients who are severely sick (suicidal, psychotic or catatonic) and rTMS could be an effective option for the rest of the patients.

\section{Durability of antidepressant effect of rTMS}

It would be worthwhile to discuss the durability of antidepressant effect of rTMS. There are only a limited number of studies that specifically investigated this question. As discussed above, there is a reasonable amount of evidence to convince oneself of the antidepressant effect of rTMS in an acute setting. Dunner et al studied the durability benefit of rTMS for over 1 year. ${ }^{37}$ They found that $62.5 \%$ of patients who had achieved remission at the end of acute treatment continued to stay in remission at the end of 1 year. However, 36.2\% of patients were given repeated sessions of rTMS within a month of completion of acute treatment. Therefore, though it appears that the response of rTMS was sustained in a majority of patients, it must be noted that these patients continued to receive antidepressants and they were given repeated sessions of rTMS as and 
when needed. ${ }^{37}$ Another meta-analysis found that the antidepressant effect got poorer with a longer follow-up period, that is, 8-16 weeks. Antidepressant effect was better sustained in patients who were non-psychotic and treatment resistant, had less severe illness, and received antidepressants. ${ }^{16}$

\section{Efficacy of maintenance rTMS after acute response in depression}

There are a few studies that have evaluated the role of rTMS as maintenance treatment for depression. ${ }^{38-41}$ Maintenance treatment is not the same as reintroduction of rTMS in case of relapse. Maintenance treatment is a regularly scheduled regimen of rTMS sessions at fixed intervals after acute treatment over a certain period of time. ${ }^{5}$ Evidence from a recent trial that patients were randomised to once a month maintenance regimen and observation only regimen did not show any statistically significant difference between the two groups at the end of a 1 year period of observation. ${ }^{40}$ Overall, there is only equivocal evidence to support use of maintenance rTMS. $^{538-41}$
rTMS in special populations with treatment-resistant depression

Literature regarding use of rTMS in the elderly population is scant, for the prime reason being lack of inclusion of elderly in such trials. ${ }^{42}$ In the review by Sabesan et al, data from three studies were compiled. ${ }^{42-45}$ Among these, two papers reported no benefit with rTMS and only one reported benefit with the help of rTMS. However, the trial which reported benefit had used higher dose, that is, around 18000 pulses for treatment. ${ }^{43}$ Sabesan et al also included data from many open-label trials and case reports/series. ${ }^{42}$ Based on their assessment, they have discussed moderators of efficacy of rTMS in the elderly. They could not find any consistent evidence that age could be a moderating factor for response to treatment. Brain atrophy, which is common with increasing age, could have had a significant effect because as the distance between scalp and cortex increases, the intensity of magnetic field reaching cortical surface decreases exponentially. In addition, atrophy is disproportionate in different brain areas and it is generally the frontal cortex which is most affected. ${ }^{46}$ The effect of dose has already been exemplified in the discussion above, where it was

\section{Table 1 Systematic reviews on efficacy of TMS in depression}

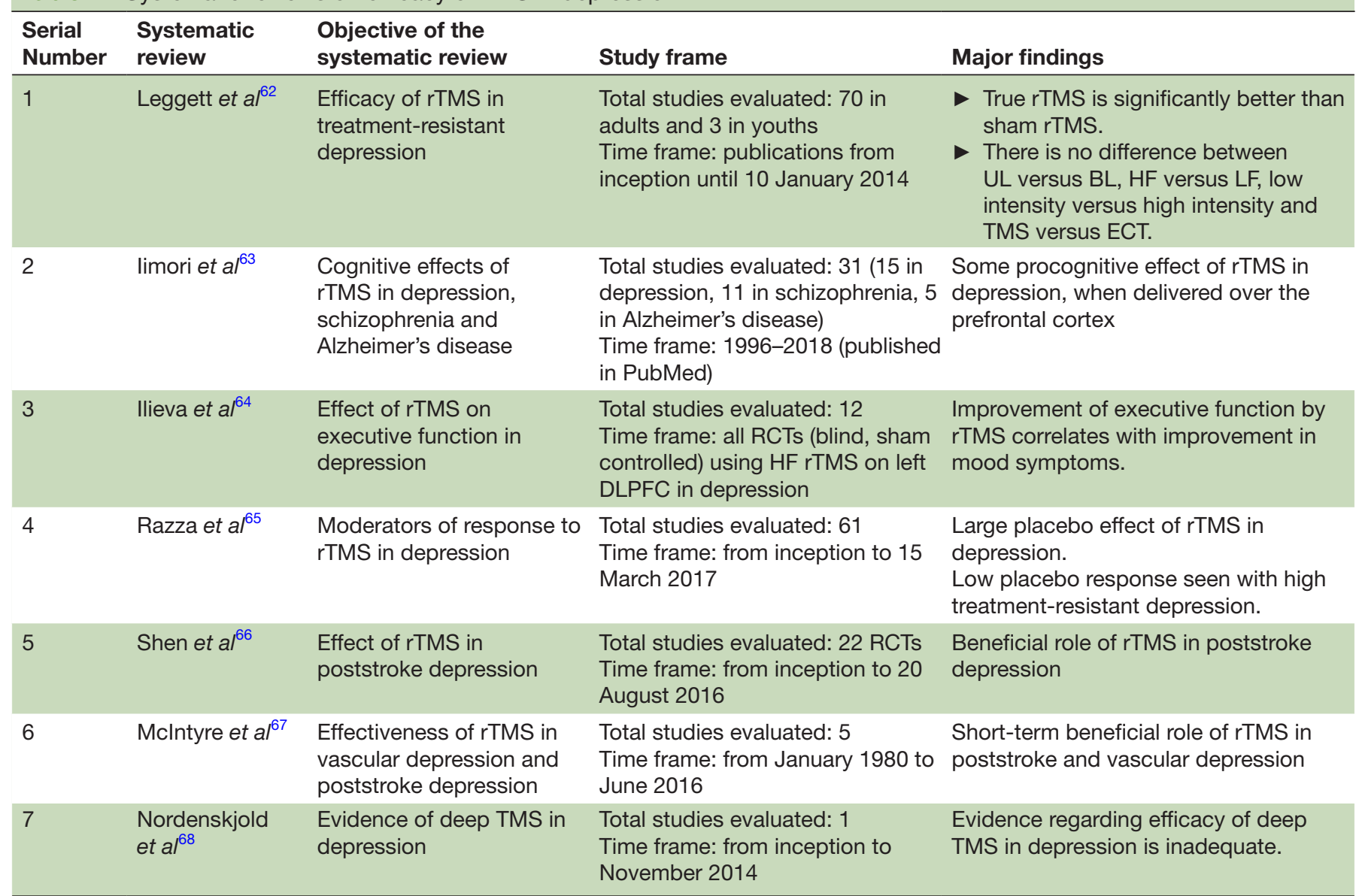

BL, bilateral; DLPFC, dorsolateral prefrontal cortex; ECT, electroconvulsive therapy; HF, high frequency; LF, low frequency; RCT, randomised controlled trial;TMS, transcranial magnetic stimulation; UL, unilateral; rTMS, repetitive TMS. 
discussed that the paper describing rTMS to be more beneficial had used a higher dose. ${ }^{43}$

There are insufficient data in the elderly group to comment if presence of treatment resistance, psychosis, cognitive impairment or medical comorbidities have any moderating effect in response to rTMS. $^{42}$ In a recent study, Trevizol $e t a l^{47}$ had evaluated the efficacy of rTMS (bilateral and high frequency unilateral on left side) in older adults with treatment-resistant depression. Patients who received bilateral sequential rTMS were reportedly to have greater rate of response $(40 \%)$ compared with unilateral and sham TMS groups. ${ }^{47}$ Hence, bilateral TMS can be a better choice to deal with treatment-resistant depression in the elderly.

Some early evidence suggest that TMS may be useful in patients with poststroke depression. ${ }^{48}$ Patients with Parkinson's disease with comorbid depression show some improvement in the motor symptoms after dTMS, however the improvement is not superior to sham TMS, as found in a recent study. ${ }^{49}$ There are few other reviews that have tried to look for effect of rTMS on cognitive profiles, executive functions and placebo response of rTMS. Other reviews have looked into the effect of rTMS in poststroke depression, vascular depression and effects of Hesel-coil TMS in depression. These reviews have been summarised in table 1.

In pregnant patients, rTMS comes with a great promise as being non-pharmacological, it could be thought of as being safe for fetuses. Safety of mother and fetus is of the utmost importance while treating any pregnant patient for any condition across medical specialties. Due to safety concerns, treatment of depressive episodes during pregnancy often becomes challenging. As a result of alterations in physiological parameters, pharmacokinetics and pharmacodynamics during pregnancy, a pseudotreatment-resistant state is often produced. Neuromodulation techniques may be useful in augmenting the therapeutic response, thereby overcoming the pseudoresistance. There have been a few trials, case reports, follow-up studies and review articles on safety and utility of rTMS in depressed pregnant patients. ${ }^{50-55}$ In a Turkish study comprising 30 depressed pregnant patients, there was a reduction in mean scores of HDRS by around 13 points after 18 sessions of rTMS given over 3 weeks. ${ }^{52}$ Overall, $20.7 \%$ of patients had remission, $34.5 \%$ had partial response and $3.4 \%$ had worsening of depression. Patients did not experience any major side effects. A review by Felipe and Ferrão gives a broader view of the issue. ${ }^{51}$ After combining data from 12 studies, they had patients who were in their various stages of pregnancy and received rTMS as monotherapy or as adjunct to antidepressant. Response rate across the studies evaluated in this review varied from $41.4 \%$ to $70 \%$. Rate of remission varied from $20.75 \%$ to $30 \%$. Response was defined on a basis of more than $50 \%$ reduction in symptom scale score. Therefore, it appears that patients had an acceptable response rate. Tolerability was also good and no major side effects were reported in pregnant patients receiving rTMS $^{51}$ Another recent study from Turkey has discussed the results of follow-up of children of mothers who had received rTMS for depression during their pregnancies. ${ }^{50}$ The children were evaluated at a mean age of 32.4 months. These children did not show any problem with motor or cognitive development. Language development was found to be poorer in a few children, but it was comparable to those who had received antidepressants during pregnancy in other studies. ${ }^{50}$ Furthermore, rTMS has also been tried in patients with postpartum depression. In one such study, nine patients with postpartum depression were treated exclusively with rTMS, of which eight achieved

Table 2 Relevance of rTMS in special populations with treatment-resistant depression

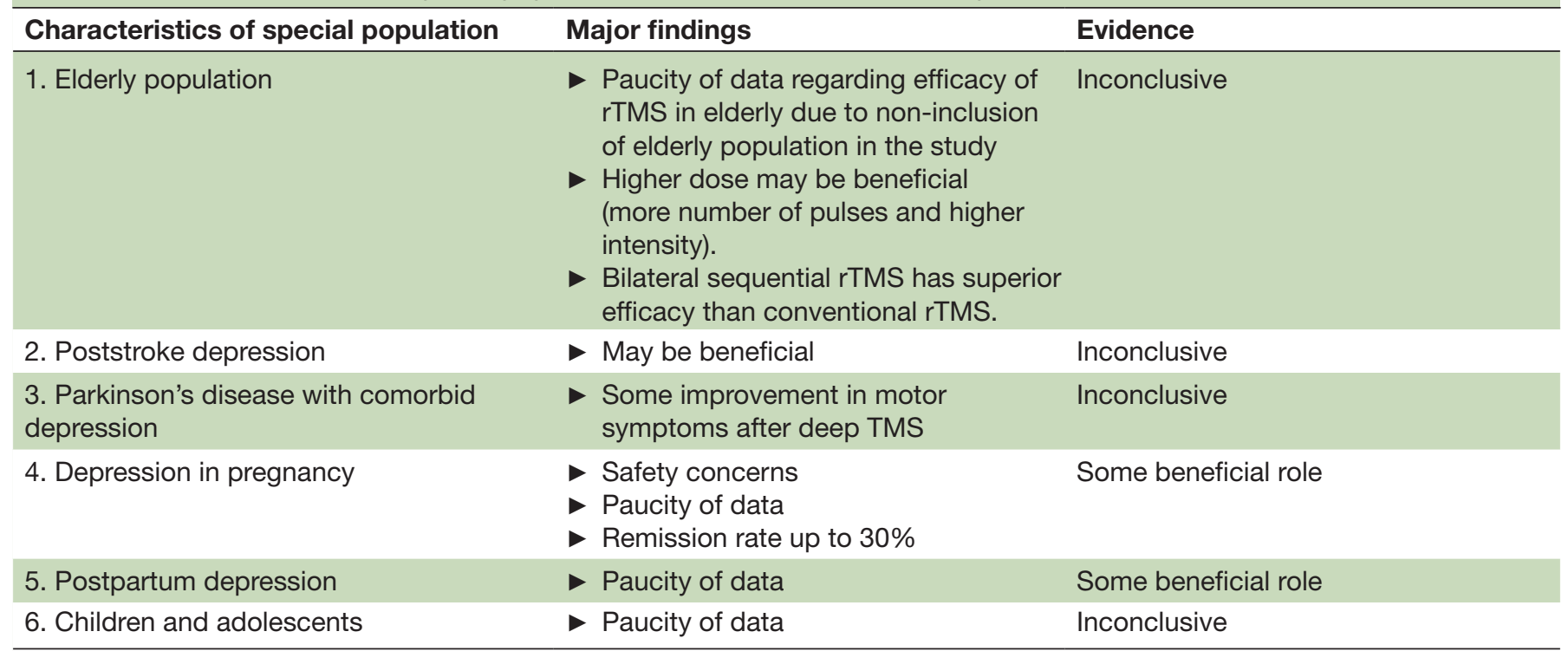

TMS, transcranial magnetic stimulation; rTMS, repetitive TMS. 
remission and seven continued to remain in remission without medications. ${ }^{56}$ As research is under progress, more data are likely to be available soon regarding utility and safety of rTMS in postpartum depression. ${ }^{57}$ Very recently, an RCT used active TMS $(n=11)$ versus sham TMS $(n=11)$ in pregnant women with depression. Low-frequency rTMS over the right DLPFC was used in the study. The response rate was found to be $81.82 \%$ in the active TMS group versus $45.45 \%$ in the sham TMS group. Remission rate was also higher in the active TMS group; however, late preterm births were reported among three women (out of 11) who were receiving active TMS. ${ }^{58}$ Studies in larger populations can give a better understanding about the safety and efficacy of TMS in pregnancy.

There is very limited literature on the efficacy of rTMS in the treatment of treatment-resistant depression in children and adolescents. Essentially, rTMS has been used across a wide range of psychiatric and neurological disorders in children and adolescents, viz autism, depression, Tourette's syndrome, perinatal stroke, epilepsy, cerebral palsy, and so forth. ${ }^{59}$ While there is a lot of discussion on the safety of rTMS in this age group, only a little has been explored regarding efficacy of the same in depression. ${ }^{59-61}$ Wall et al evaluated 18 patients with depression who were given rTMS and found the same to be effective and safe in terms of neurocognitive side effects. ${ }^{61}$ Sufficient data are not available to make any further comment. Table 2 summarises the evidence of rTMS in treating treatment-resistant depression in special group populations.

\section{CONCLUSION}

Furthermore, rTMS is rapidly gaining popularity as a treatment modality for depression. There is growing evidence to support its use in patients with depression as a monotherapy or as adjunct with pharmacotherapy. Additionally, rTMS has been found to be safe and effective in pregnant patients and elderly patients, that use of either pharmacotherapy or ECT is challenging because of possible risk of adverse reactions. While there is growing evidence to support its use in an acute setting, there is limited literature to support long-term benefits of the same. The day is not far when rTMS shall be practised routinely for treatment. To be able to achieve this, more research is needed in order to bring out standardised protocols of administration of rTMS covering issues like localisation, frequency, intensity, number of pulses, maintenance regimen, unilateral or bilateral mode, concurrent medication, and so forth. This may lead to delivery of highest efficacy treatment with least possible side effects.

Contributors Both authors are involved equally in conceptualising, literature review, developing the manuscript and editing the manuscript.

Funding The authors have not declared a specific grant for this research from any funding agency in the public, commercial or not-for-profit sectors.

Competing interests None declared.
Patient consent for publication Not required.

Provenance and peer review Not commissioned; externally peer reviewed.

Data availability statement Reserach can be assessed on PubMed database.

Open access This is an open access article distributed in accordance with the Creative Commons Attribution Non Commercial (CC BY-NC 4.0) license, which permits others to distribute, remix, adapt, build upon this work non-commercially, and license their derivative works on different terms, provided the original work is properly cited, appropriate credit is given, any changes made indicated, and the use is non-commercial. See: http://creativecommons.org/licenses/by-nc/4.0/.

\section{REFERENCES}

1 WHO Fact Sheet on Depression. Secondary who fact sheet on depression, 2018. Available: http://www.who.int/news-room/factsheets/detail/depression

2 Cipriani A, Barbui C, Butler R, et al. Depression in adults: drug and physical treatments. BMJ Clin Evid 2011;2011. [Epub ahead of print: 25 May 2011].

3 Pradhan B, Parikh T, Makani R, et al. Ketamine, transcranial magnetic stimulation, and depression specific yoga and mindfulness based cognitive therapy in management of treatment resistant depression: review and some data on efficacy. Depress Res Treat 2015;2015:1-14.

4 Mrazek DA, Hornberger JC, Altar CA, et al. A review of the clinical, economic, and societal burden of treatment-resistant depression: 1996-2013. PS 2014;65:977-87.

5 Guo Q, Li C, Wang J. Updated review on the clinical use of repetitive transcranial magnetic stimulation in psychiatric disorders. Neurosci Bull 2017;33:747-56.

6 Klomjai W, Katz R, Lackmy-Vallée A. Basic principles of transcranial magnetic stimulation (TMS) and repetitive TMS (rTMS). Ann Phys Rehabil Med 2015;58:208-13.

7 Machado S, Arias-Carrion O, Paes F, et al. Repetitive transcranial magnetic stimulation for clinical applications in neurological and psychiatric disorders: an overview. EAJM 2013;45:191-206.

8 Barker AT, Jalinous R, Freeston IL. Non-Invasive magnetic stimulation of human motor cortex. The Lancet 1985;325:1106-7.

9 Chen R, Classen J, Gerloff C, et al. Depression of motor cortex excitability by low-frequency transcranial magnetic stimulation. Neurology 1997;48:1398-403.

10 Chung SW, Hoy KE, Fitzgerald PB. Theta-burst stimulation: a new form of TMS treatment for depression? Depress Anxiety 2015;32:182-92.

11 Huang Y-Z, Edwards MJ, Rounis E, et al. Theta burst stimulation of the human motor cortex. Neuron 2005;45:201-6.

12 Blumberger DM, Vila-Rodriguez F, Thorpe KE, et al. Effectiveness of theta burst versus high-frequency repetitive transcranial magnetic stimulation in patients with depression (THREE-D): a randomised non-inferiority trial. The Lancet 2018;391:1683-92.

13 Grimm S, Beck J, Schuepbach D, et al. Imbalance between left and right dorsolateral prefrontal cortex in major depression is linked to negative emotional judgment: an fMRI study in severe major depressive disorder. Biol Psychiatry 2008;63:369-76.

14 George MS, Nahas Z, Molloy M, et al. A controlled trial of daily left prefrontal cortex TMS for treating depression. Biol Psychiatry 2000;48:962-70.

15 Klein E, Kreinin I, Chistyakov A, et al. Therapeutic efficacy of right prefrontal slow repetitive transcranial magnetic stimulation in major depression. Arch Gen Psychiatry 1999;56:315-20.

16 Kedzior KK, Reitz SK, Azorina V, et al. Durability of the antidepressant effect of the high-frequency repetitive transcranial magnetic stimulation (rTMS) in the absence of maintenance treatment in major depression: a systematic review and metaanalysis of 16 double-blind, randomized, SHAM-CONTR. Depress Anxiety 2015;32:193-203.

17 Berlim MT, Van den Eynde F, Jeff Daskalakis Z. Clinically meaningful efficacy and acceptability of low-frequency repetitive transcranial magnetic stimulation (rTMS) for treating primary major depression: a meta-analysis of randomized, double-blind and sham-controlled trials. Neuropsychopharmacology 2013;38:543-51.

18 Berlim MT, van den Eynde F, Tovar-Perdomo S, et al. Response, remission and drop-out rates following high-frequency repetitive transcranial magnetic stimulation (rTMS) for treating major depression: a systematic review and meta-analysis of randomized, double-blind and sham-controlled trials. Psychol Med 2014;44:225-39.

19 Chen J, Zhou C, Wu B, et al. Left versus right repetitive transcranial magnetic stimulation in treating major depression: a meta-analysis of randomised controlled trials. Psychiatry Res 2013;210:1260-4. 
20 Gaynes BN, Lloyd SW, Lux L, et al. Repetitive transcranial magnetic stimulation for treatment-resistant depression: a systematic review and meta-analysis. J Clin Psychiatry 2014;75:477-89.

21 Wei Y, Zhu J, Pan S, et al. Meta-Analysis of the efficacy and safety of repetitive transcranial magnetic stimulation (rTMS) in the treatment of depression. Shanghai Arch Psychiatry 2017;29:328-42.

22 Ontario HQ. Repetitive transcranial magnetic stimulation for treatment-resistant depression: a systematic review and metaanalysis of randomized controlled trials. Ont Health Technol Assess Ser 2016;16:1-66.

23 Blumberger DM, Mulsant BH, Fitzgerald $\mathrm{PB}$, et al. A randomized double-blind sham-controlled comparison of unilateral and bilatera repetitive transcranial magnetic stimulation for treatment-resistant major depression. World J Biol Psychiatry 2012;13:423-35.

24 Zhang YQ, Zhu D, Zhou XY, et al. Bilateral repetitive transcranial magnetic stimulation for treatment-resistant depression: a systematic review and meta-analysis of randomized controlled trials. Braz J Med Biol Res 2015;48:198-206.

25 Berlim MT, Van den Eynde F, Daskalakis ZJ. A systematic review and meta-analysis on the efficacy and acceptability of bilateral repetitive transcranial magnetic stimulation (rTMS) for treating major depression. Psychol Med 2013;43:2245-54.10.1017/ S0033291712002802

26 Stubbeman WF, Zarrabi B, Bastea S, et al. Bilateral neuronavigated $20 \mathrm{~Hz}$ theta burst TMS for treatment refractory depression: An open label study. Brain Stimul 2018;11:953-5.

27 Rapinesi C, Bersani FS, Kotzalidis GD, et al. Maintenance deep transcranial magnetic stimulation sessions are associated with reduced depressive relapses in patients with unipolar or bipolar depression. Front Neurol 2015;6:16.

28 Tavares DF, Myczkowski ML, Alberto RL, et al. Treatment of bipolar depression with deep TMS: results from a doubleblind, randomized, parallel group, sham-controlled clinical trial. Neuropsychopharmacology 2017;42:2593-601.

$29 \mathrm{SH} \mathrm{H}$, Lai JB, DR X, et al. Efficacy of repetitive transcranial magnetic stimulation with quetiapine in treating bipolar II depression: a randomized, double-blinded, control study. Sci Rep 2016;6.

30 Dubin MJ, llieva IP, Deng Z-D, et al. A double-blind pilot dosing study of low field magnetic stimulation (LFMS) for treatment-resistant depression (TRD). J Affect Disord 2019;249:286-93.

31 Baeken C. Accelerated rTMS: a potential treatment to alleviate refractory depression. Front Psychol 2017;2018.

32 Schulze L, Feffer K, Lozano C, et al. Number of pulses or number of sessions? an open-label study of trajectories of improvement for once-vs. twice-daily dorsomedial prefrontal rTMS in major depression. Brain Stimul 2018;11:327-36.

33 Donse L, Padberg F, Sack AT, et al. Simultaneous rTMS and psychotherapy in major depressive disorder: clinical outcomes and predictors from a large naturalistic study. Brain Stimul 2018;11:337-45

34 Hansen PEB, Ravnkilde B, Videbech P, et al. Low-Frequency repetitive transcranial magnetic stimulation inferior to electroconvulsive therapy in treating depression. J Ect 2011;27:26-32

35 Berlim MT, Van den Eynde F, Daskalakis ZJ. Efficacy and acceptability of high frequency repetitive transcranial magnetic stimulation (rTMS) versus electroconvulsive therapy (ECT) for major depression: a systematic review and meta-analysis of randomized trials. Depress Anxiety 2013;30:614-23.

36 Ren J, Li H, Palaniyappan L, et al. Repetitive transcranial magnetic stimulation versus electroconvulsive therapy for major depression: a systematic review and meta-analysis. Prog Neuropsychopharmacol Biol Psychiatry 2014;51:181-9.

37 Dunner DL, Aaronson ST, Sackeim HA, et al. A multisite, naturalistic, observational study of transcranial magnetic stimulation for patients with pharmacoresistant major depressive disorder: durability of benefit over a 1-year follow-up period. J Clin Psychiatry 2014;75:1394-401

38 Fitzgerald PB, Grace N, Hoy KE, et al. An open label trial of clustered maintenance rTMS for patients with refractory depression. Brain Stimul 2013;6:292-7.

39 O'Reardon JP, Blumner KH, Peshek AD, et al. Long-Term maintenance therapy for major depressive disorder with rTMS. J Clin Psychiatry 2005;66:1524-8.

40 Philip NS, Dunner DL, Dowd SM, et al. Can medication free, treatment-resistant, depressed patients who initially respond to TMS be maintained off medications? A prospective, 12-month multisite randomized pilot study. Brain Stimul 2016;9:251-7.

41 Richieri R, Guedj E, Michel P, et al. Maintenance transcranial magnetic stimulation reduces depression relapse: a propensityadjusted analysis. J Affect Disord 2013;151:129-35.
42 Sabesan P, Lankappa S, Khalifa N, et al. Transcranial magnetic stimulation for geriatric depression: promises and pitfalls. World $\mathrm{J}$ Psychiatry 2015;5:170-81.

43 Jorge RE, Moser DJ, Acion L, et al. Treatment of vascular depression using repetitive transcranial magnetic stimulation. Arch Gen Psychiatry 2008;65:268-76.

44 Manes F, Jorge R, Morcuende M, et al. A controlled study of repetitive transcranial magnetic stimulation as a treatment of depression in the elderly. Int. Psychogeriatr. 2001;13:225-31.

45 Mosimann UP, Schmitt W, Greenberg BD, et al. Repetitive transcranial magnetic stimulation: a putative add-on treatment for major depression in elderly patients. Psychiatry Res 2004;126:123-33.10.1016/j.psychres.2003.10.006

46 McConnell KA, Nahas Z, Shastri A, et al. The transcranial magnetic stimulation motor threshold depends on the distance from coil to underlying cortex: a replication in healthy adults comparing two methods of assessing the distance to cortex. Biol Psychiatry 2001;49:454-9.

47 Trevizol AP, Goldberger KW, Mulsant BH, et al. Unilateral and bilateral repetitive transcranial magnetic stimulation for treatment-resistant late-life depression. Int J Geriatr Psychiatry 2019;34:822-7.

48 Duan X, Yao G, Liu Z, et al. Mechanisms of transcranial magnetic stimulation treating on post-stroke depression. Front Hum Neurosci 2018;12:215

49 Cohen OS, Rigbi A, Yahalom G, et al. Repetitive deep TMS for Parkinson disease: a 3-month double-blind, randomized shamcontrolled study. J Clin Neurophysiol 2018;35:159-65.

50 Eryılmaz G, Hızlı Sayar G, Özten E, et al. Follow-Up study of children whose mothers were treated with transcranial magnetic stimulation during pregnancy: preliminary results. Neuromodulation 2015;18:255-60.

51 Felipe RdeM, Ferrão YA, UFCSPA, Brazil. Transcranial magnetic stimulation for treatment of major depression during pregnancy: a review. Trends Psychiatry Psychother. 2016;38:190-7.

52 Hızlı Sayar G, Ozten E, Tufan E, et al. Transcranial magnetic stimulation during pregnancy. Arch Womens Ment Health 2014;17:311-5.

53 Klirova M, Novak T, Kopecek M, et al. Repetitive transcranial magnetic stimulation (rTMS) in major depressive episode during pregnancy. Neuro Endocrinol Lett 2008;29:69-70.

54 Zhang D, Hu Z. Rtms may be a good choice for pregnant women with depression. Arch Womens Ment Health 2009;12:189-90.

55 Zhang X, Liu K, Sun J, et al. Safety and feasibility of repetitive transcranial magnetic stimulation (rTMS) as a treatment for major depression during pregnancy. Arch Womens Ment Health 2010;13:369-70.

56 Garcia KS, Flynn P, Pierce KJ, et al. Repetitive transcranial magnetic stimulation treats postpartum depression. Brain Stimul 2010;3:36-41.

57 Andriotti T, Stavale R, Nafee T, et al. ASSERT trial - How to assess the safety and efficacy of a high frequency rTMS in postpartum depression? A multicenter, double blinded, randomized, placebocontrolled clinical trial. Contemp Clin Trials Commun 2017;5:86-91.

58 Kim DR, Wang E, McGeehan B, et al. Randomized controlled trial of transcranial magnetic stimulation in pregnant women with major depressive disorder. Brain Stimul 2019;12:96-102.

59 Hameed MQ, Dhamne SC, Gersner R, et al. Transcranial magnetic and direct current stimulation in children. Curr Neurol Neurosci Rep 2017;17:11

60 Krishnan C, Santos L, Peterson MD, et al. Safety of noninvasive brain stimulation in children and adolescents. Brain Stimul 2015;8:76-87.

61 Wall CA, Croarkin PE, McClintock SM, et al. Neurocognitive effects of repetitive transcranial magnetic stimulation in adolescents with major depressive disorder. Front Psychiatry 2013;4.

62 Leggett LE, Soril LJ, Coward S, et al. Repetitive transcranial magnetic stimulation for treatment-resistant depression in adult and youth populations: a systematic literature review and meta-analysis. Prim Care Companion CNS Disord 2015;17.

63 limori T, Nakajima S, Miyazaki T, et al. Effectiveness of the prefrontal repetitive transcranial magnetic stimulation on cognitive profiles in depression, schizophrenia, and Alzheimer's disease: a systematic review. Prog Neuropsychopharmacol Biol Psychiatry 2019;88:31-40.

64 Ilieva IP, Alexopoulos GS, Dubin MJ, et al. Age-Related repetitive transcranial magnetic stimulation effects on executive function in depression: a systematic review. Am J Geriatr Psychiatry 2018;26:334-46

65 Razza LB, Moffa AH, Moreno ML, et al. A systematic review and meta-analysis on placebo response to repetitive transcranial magnetic stimulation for depression trials. Prog Neuropsychopharmacol Biol Psychiatry 2018;81:105-13. 
66 Shen X, Liu M, Cheng Y, et al. Repetitive transcranial magnetic stimulation for the treatment of post-stroke depression: a systematic review and meta-analysis of randomized controlled clinical trials. J Affect Disord 2017;211:65-74.

67 Mclntyre A, Thompson S, Burhan A, et al. Repetitive transcranial magnetic stimulation for depression due to cerebrovascular disease: a systematic review. J Stroke Cerebrovasc Dis 2016;25:2792-800.

68 Nordenskjöld A, Mårtensson B, Pettersson A, et al. Effects of Hesel-coil deep transcranial magnetic stimulation for depression - a systematic review. Nord J Psychiatry 2016;70:492-7.

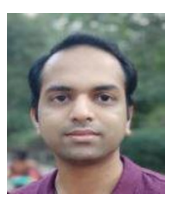

Dr. Aditya Somani, MD, DNB, MNAMS, completed his graduation at Maulana Azad Medical College, New Delhi, India in the year 2009 and post-graduation in Psychiatry at King George's Medical University, Lucknow, India in the year 2014. He was admitted to the degree of DNB in Psychiatry by National Board of Examination, New Delhi in 2017. He is also a member of National Academy of Medical Sciences, New Delhi. He is currently working as Deputy Medical Superintendent at Mental Health Institute, Chandigarh, India, since Sept 2018 and primarily takes care of administrative affairs of the institute along with teaching and research activities. His research interests include biological psychiatry and consultation-liaison psychiatry. 